\title{
The effects of mesenchymal stem cells injected via different routes on modified IL-12-mediated antitumor activity
}

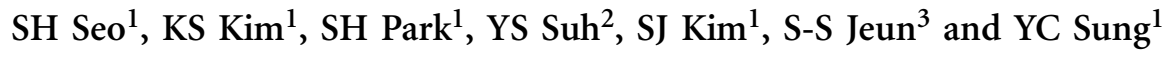 \\ Owing to its tumor tropism and prolonged transgene expression, mesenchymal stem cell (MSC) has been considered as an \\ ideal delivery vehicle for cancer gene therapies or therapeutic vaccines. In this study, we demonstrated that intratumoral (i.t.) \\ injection of MSCs expressing modified interleukin-12 (MSCs/IL-12M) exhibited stronger tumor-specific T-cell responses and \\ antitumor effects as well as more sustained expressions of IL-12 and interferon (IFN)- $\gamma$ in both sera and tumor sites than did \\ IL-12M-expressing adenovirus ( $\mathrm{rAd} / \mathrm{IL}-12 \mathrm{M}$ ) in mice bearing both solid and metastatic tumors. Subcutaneous (s.c.) injection of \\ MSCs/IL-12M at contralateral site of tumor exhibited similar levels of serum IL-12 and IFN- $\gamma$ as i.t. injection, but much weaker \\ antitumor effects in both B16F10 melanoma and TC-1 cervical cancer models than i.t. injection. Although intravenous (i.v.) \\ injection elicited earlier peak serum levels of cytokines, it induced weaker tumor-specific T-cell responses and antitumor \\ effects than i.t. injection, indicating that serum cytokine levels are not surrogate indicators of antitumor effects. Taken together, \\ these results indicated that MSC is more efficient than adenovirus as a cytokine gene delivery vehicle and that i.t. injection of \\ MSCs/IL-12M is the best approach to induce strong tumor-specific T-cell responses that correlate with anti-metastatic effects \\ as well as inhibition of solid tumor growth, although MSCs themselves have an ability to migrate into the tumor site. In addition, \\ MSCs/IL-12M embedded in Matrigel (MSCs/IL-12M/Matrigel) exhibited significant antitumor effects even in immunodeficient \\ mice such as SCID and BNX mice lacking T, B and natural killer (NK) cells, but not in IFN- $\gamma$ knockout mice. Our findings \\ provide an optimal approach for designing an efficient clinical protocol of MSC-based cytokine gene therapy to induce strong \\ tumor-specific T-cell responses and therapeutic anticancer efficacy.
}

Gene Therapy (2011) 18, 488-495; doi:10.1038/gt.2010.170; published online 13 January 2011

Keywords: mesenchymal stem cell; interleukin-12; metastasis; injection route; Matrigel

\section{INTRODUCTION}

Over the past several decades, chemotherapeutic agents and radiationbased therapies have provided significant benefits and cures by eliminating tumor mass. However, current therapies appear to cause significant systemic or local toxicity. ${ }^{1}$ In addition, the incidence of cancer relapse due to micro-metastasis and/or the generation of tumors resistant to current therapies cause many complications, ${ }^{2}$ suggesting that novel approaches or modifications of current protocols are needed to overcome these hurdles. Immunotherapy, as a new direction in cancer therapy, is based on utilizing our immune system or its components to attack cancer cells with high specificity. In the context of cancer immunotherapy, cytokines have been widely utilized as means of boosting both innate and adaptive immune responses against tumor. However, systemic in vivo distribution of recombinant cytokine proteins is frequently associated with serious and even lifethreatening consequences as well as marginal clinical responses in most patients. ${ }^{3,4}$ As a result, several delivery vehicles for example, adenoviral vector, plasmid DNA or mesenchymal stem cells (MSCs) have been utilized to target cytokine genes and express them in the area of interest. ${ }^{5-7}$

Intratumoral delivery of plasmid DNA encoding cytokines such as IL-12 was effective in tumor regression by inducing $\mathrm{CD}^{+} \mathrm{T}$-cell infiltration and cytotoxic T lymphocyte activity. ${ }^{6}$ In the case of using an adenovirus as a delivery vehicle, the efficiency of cytokine gene delivery into a tumor may vary depending on the expression level of coxsackievirus and adenovirus receptor on tumor. ${ }^{8}$ In addition, preexisting neutralizing antibody or neutralizing antibody generated by repeated injection of rAd vector could hinder the therapeutic efficacy of transgene. ${ }^{9}$ Recently, it was reported that the use of ex vivo rAdtransduced MSCs can avoid the current obstacles present in adenovirus-based gene therapy and that MSCs expressing IL-12 exerted stronger antitumor activity than did plasmid DNA encoding IL-12. ${ }^{10}$ However, it is still not clear whether the antitumor effect as well as T-cell responses induced by cytokine-expressing MSCs is affected by injection route.

Bone marrow-derived MSCs are adult pluripotent progenitor cells with the potential to differentiate into osteoblasts, chondrocytes or adipocytes. ${ }^{11-13}$ Furthermore, MSCs expressing transgenes maintained long-term expression (up to 6 months) in vivo because of their hypoimmunogenic properties and production of immunosuppressive molecules. ${ }^{14,15}$ More importantly, MSCs were shown to migrate into inflamed, damaged tissues and tumor sites, ${ }^{13,16}$ which make them ideal cellular vehicles because of their capability to deliver cytokine genes directly to tumors compared with other cellular delivery vehicles such as fibroblasts, dendritic cells (DCs) and autologous tumor cells. Administration of genetically modified MSCs producing interferon

${ }^{1}$ Division of Molecular and Life Science, Pohang University of Science and Technology (POSTECH), Pohang, Gyeongbuk, Republic of Korea; ${ }^{2}$ Research Institute, Genexine Co., Ltd, \#206, POSTECH Biotech. Center, Pohang, Gyeongbuk, Republic of Korea and ${ }^{3}$ Department of Biomedical Science, Catholic University of Korea, Seoul, South Korea Correspondence: Dr YC Sung, Division of Molecular and Life Sciences, Integrative Bioscience \& Biotechnology, WCU, Pohang University of Science and Technology (POSTECH), San 31, Hyoja-Dong, Nam-gu, Pohang, Kyungbuk 790-784, Republic of Korea.

E-mail: ycsung@postech.ac.kr

Received 27 July 2010; revised 7 October 2010; accepted 15 October 2010; published online 13 January 2011 
(IFN)- $\alpha$, IFN- $\beta$ or tumor necrosis factor-related apoptosis-inducing ligand via intravenous (i.v.) route reduced the incidence of lung metastasis in mouse and human cancer models. ${ }^{16-19}$ In addition, i.v. injected IL-12-expressing MSCs (MSCs/IL-12) inhibited the metastasis of lymphatic tumors without causing severe adverse effects. ${ }^{7}$

IL-12, comprised of the p35 and p40 subunits, is a pro-inflammatory cytokine produced by antigen-presenting cells and induces proliferation of natural killer (NK) cells and cytotoxic T lymphocytes as well as IFN- $\gamma$ production. ${ }^{20}$ IL-12-induced IFN- $\gamma$ was shown to upregulate major histocompatibility complexes and adhesion molecules, resulting in enhanced susceptibility of tumor cell to cytotoxic T lymphocytemediated killing. ${ }^{21,22}$ In addition, IFN- $\gamma$ suppresses angiogenesis through the production of IFN-inducible protein-10 and monokine induced by IFN- $\gamma .{ }^{23,24}$ Owing to its diverse effects, IL- 12 has been regarded as a master regulator of antitumor immunity among many cytokines. ${ }^{25-27}$ However, there is a major obstacle in IL-12 gene-based immunotherapy because of its co-expression of the 440 subunit which is known as a natural antagonist of IL- $12 .{ }^{28}$ As previously reported, ${ }^{29}$ modified IL-12 (IL-12M), an N-glycosylation mutant of IL-12 at Asn220, selectively reduces secretion of the p40 subunit and thus significantly enhances co-delivered antigen-specific T-cell responses. In addition, there are no significant differences in biological activity in terms of IFN- $\gamma$ induction and receptor-binding affinity between IL-12 and IL-12M.

In this study, we demonstrated for the first time that intratumoral (i.t.) injection of IL-12M-expressing MSCs exhibited the strongest antitumor effects compared with other injection routes (i.v. or subcutaneous; s.c.), which closely correlated with tumor-specific T-cell responses but not with serum cytokine levels in mice bearing both solid and metastatic tumor. In addition, Matrigel embedding of
MSCs/IL-12M exhibited significant anti-metastatic effect as well as inhibition of solid tumor growth even in immunodeficient mice.

\section{RESULTS}

MSCs/IL-12M inhibited both pulmonary metastasis and solid tumor growth at a greater degree than did rAd/IL-12M

Rat bone marrow-derived MSCs were transduced with $\mathrm{rAd} / \mathrm{IL}-12 \mathrm{M}$ using the tetrameric form of cell-permeable peptide (4HP4) to generate MSCs/IL-12M as previously described ${ }^{30}$ and $>95 \%$ of MSCs were transduced (data not shown). The inclusion of 4HP4 significantly enhanced transduction efficiency of rAd/IL-12M into MSCs, resulting in higher IL-12 expression level by more than 30 -fold (data not shown). In order to assess the relative therapeutic efficacy of $\mathrm{rAd} / \mathrm{IL}-12 \mathrm{M}$ and MSCs/IL-12M, mice bearing both solid and metastatic tumors were generated by injecting with B16F10 s.c. and then i.v. At 1 and 8 days after i.v. tumor injection, tumor-bearing mice were either left untreated or injected with MSCs, $\mathrm{rAd} / \mathrm{IL}-12 \mathrm{M}$ or MSCs/IL-12M into solid tumor.

There were no significant differences in solid tumor progression, the number of lung metastases and survival rates between mice injected with MSCs and untreated mice, indicating that MSCs alone had no effect on tumor growth (Figure 1a-c). This result is inconsistent with the previous reports that MSCs could inhibit tumor growth directly by secreting dickkopf- $1^{31}$ or promote tumor growth by suppressing immune cells through release of anti-inflammatory molecules such as transforming growth factor- $\beta$, IL-10 and prostaglandin E2. ${ }^{32}$ As expected, i.t. injection of $\mathrm{rAd} / \mathrm{IL}-12 \mathrm{M}$ inhibited both solid tumor growth and the formation of lung metastases, but the effects were much lower than those of MSCs/IL-12M (Figures 1a, b; $P<0.01)$. All mice treated with $\mathrm{rAd} / \mathrm{IL}-12 \mathrm{M}$ delayed their survival,
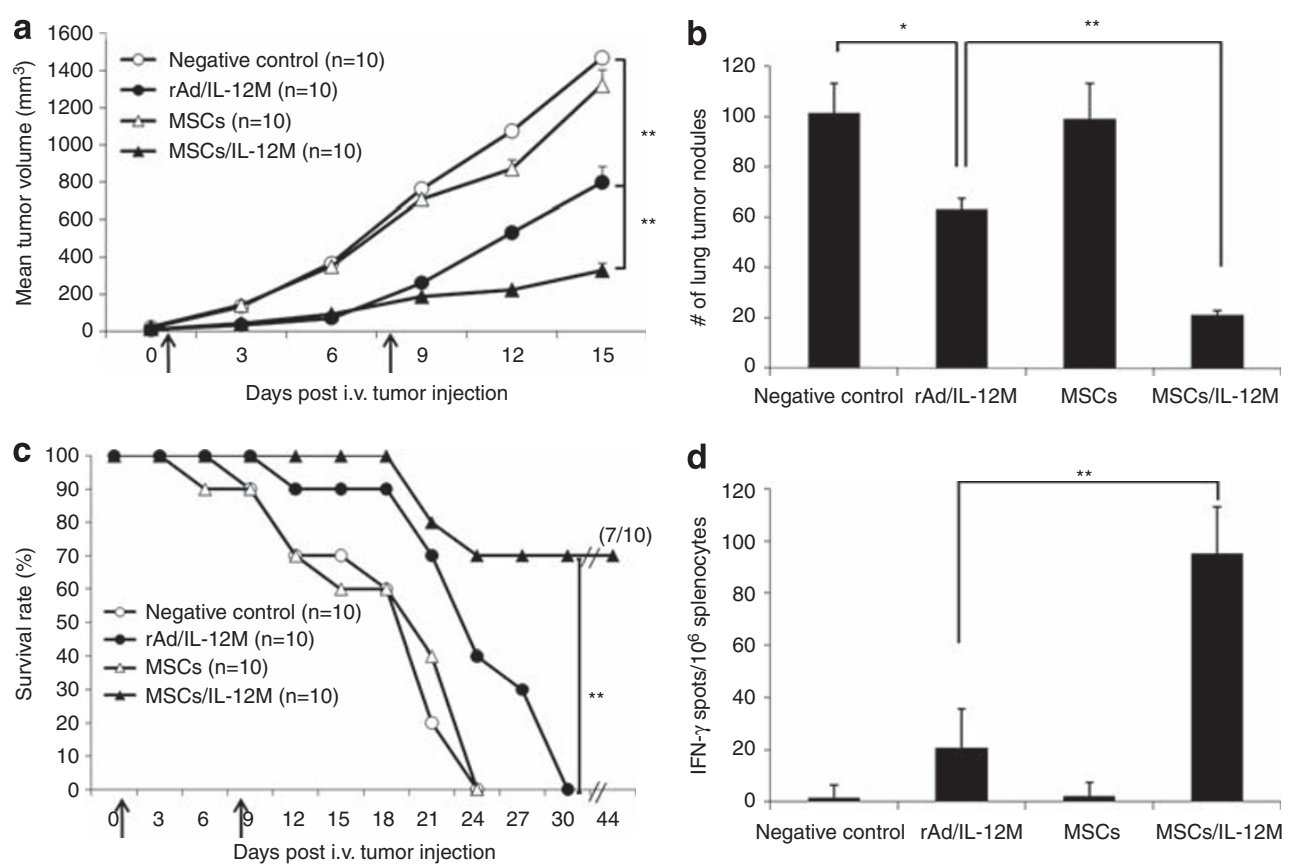

Figure 1 Relative antitumor activities between mesenchymal stem cell (MSCs) expressing modified interleukin-12 (MSCs/IL-12M) and rAd/IL-12M. When subcutaneous (s.c.) injected B16F10 tumor cells grew up to a size of about $5 \mathrm{~mm}$ in diameter, mice were injected with $2 \times 10^{5}$ cells of B16F10 cells i.v. At day 1 and 8 after intravenous (i.v.) injection, the tumor-bearing mice were treated with $5 \times 10^{6}$ p.f.u. of rAd/IL- $12 \mathrm{M}, 1 \times 10^{5} \mathrm{MSCs}$ or MSCs/LL-12M as indicated by arrows. Tumor mass progression (a) and survival rates (c) were monitored at 3-day intervals. Lung tumor nodules were assessed 14 days after i.v. tumor injection (b). At 14 days after i.v. tumor injection, splenocytes were harvested and the frequency of tumor-specific interferon (IFN)- $\gamma$-producing cells per $10^{6}$ splenocytes was determined by an IFN- $\gamma$ enzyme-linked immunosorbent spot (ELISPOT) assay (d). These are representative of three independent experiments with similar results. ${ }^{*} P<0.05$ and ${ }^{*} P<0.01$. 
but died 30 days after i.v. tumor injection. In contrast, 70\% of the MSCs/IL-12M-treated mice survived at the end of experiment (Figure $1 c, P<0.01$ ). It is worth noting that mice bearing both solid and metastatic tumor died when solid tumor volume reached $400 \mathrm{~mm}^{3}$. As mortality in mice bearing solid tumor only was observed when tumor volume was $>1000 \mathrm{~mm}^{3}$, (see ref. 5), metastatic tumor might be a major cause of death in this study.

When tumor-specific T-cell responses were assessed by IFN- $\gamma$ enzyme-linked immunosorbent spot assay using tumor lysate, i.t. injection of MSCs/IL-12M induced $\sim$ five-fold higher numbers of B16F10 tumor-specific IFN- $\gamma$-producing T cells than those induced by rAd/IL-12M (Figure 1d, $P<0.01$ ). Like untreated negative control, mice injected with MSCs did not develop any detectable level of tumor-specific T-cell responses.

MSCs/IL-12M appeared to elicit higher and more sustained expressions of IL-12 and IFN- $\gamma$ in serum (Figure $2 \mathrm{a}, \mathrm{b}$ ) and tumor site (Figure $2 \mathrm{c}, \mathrm{d}$ ) than rAd/IL-12M. As the same amount of rAd/IL-12M was used in ex vivo generation of MSCs/IL-12M, the difference in the level of cytokines observed here may be caused by higher ex vivo transduction efficiency of $\mathrm{rAd} / \mathrm{IL}-12 \mathrm{M}$ into MSCs by the presence of $4 \mathrm{HP} 4$ than direct in vivo infection of $\mathrm{rAd} / \mathrm{IL}-12 \mathrm{M}$ delivered via i.t. route. Interestingly, cytokines produced by i.t. injection of MSCs/IL-12M are detectable in the tumor sites for a longer time than in serum (10-14 versus 5 days). In addition, these enhanced and prolonged expressions of cytokines seem to be closely associated with the induction of strong tumor-specific T-cell responses, leading to increased antitumor effects.

Intratumoral injection of MSCs/IL-12M induced the most potent antitumor activities compared with other routes of injection

To investigate the relative effect of injection route on antitumor activity, MSCs/IL-12M were injected into mice bearing solid and metastatic B16F10 melanoma via i.t., s.c. or i.v. route. Administration of MSCs/IL-12M via an i.t. route elicited the most significant retardation of solid tumor growth and prolonged survival compared with other injection routes (s.c. and i.v.) (Figure 3A). It is worth noting that contralateral s.c. injection of MSCs/IL-12M exhibited marginal antitumor activities in terms of suppression of solid tumor growth and enhancement of survival, which agrees well with a previous report. ${ }^{33}$ Also, mice injected with MSCs/IL-12M via i.t. route showed less number of metastatic nodules than that of contralateral s.c. or i.v. route (Figure 3B). In addition, i.t. injection generated the highest frequency of tumor-specific $\mathrm{T}$ cells, followed by i.v. injection (Figure 3C), indicating that antitumor effects of MSCs/ IL-12M are closely correlated with tumor-specific T-cell responses. Similar results were observed in TC-1 cervical cancer model in terms of the induction of tumor-specific T-cell responses and antitumor effects using the same injection route for MSCs/IL-12M treatment, indicating tumor-type independent effects (Supplementary Figure 1a-d).

When serum cytokine levels were evaluated at various time points from day 1 to 7 , injection of MSCs/IL-12M via i.v. route generated earlier peak levels of IL-12 and IFN- $\gamma$ compared with other injection routes, but the relative level of these cytokines were reversed at day 5 (Figure 3D). There were no significant differences in cytokine levels and expression patterns produced by MSCs/IL-12M treatment between i.t. and s.c. routes, indicating that the level of serum cytokines is not an indicator of antitumor potential. It is worth noting that the concentration of IFN- $\gamma$ in serum follows the same kinetics as IL-12 which is not consistent to the previous report. ${ }^{34}$ The discrepancy may be due to difference in assay condition. As we started to check the levels of IFN- $\gamma$ at 1 day after treatment then every 2 days thereafter, delayed expression of IFN- $\gamma$ may not be reflected in this study as previous report. ${ }^{35}$
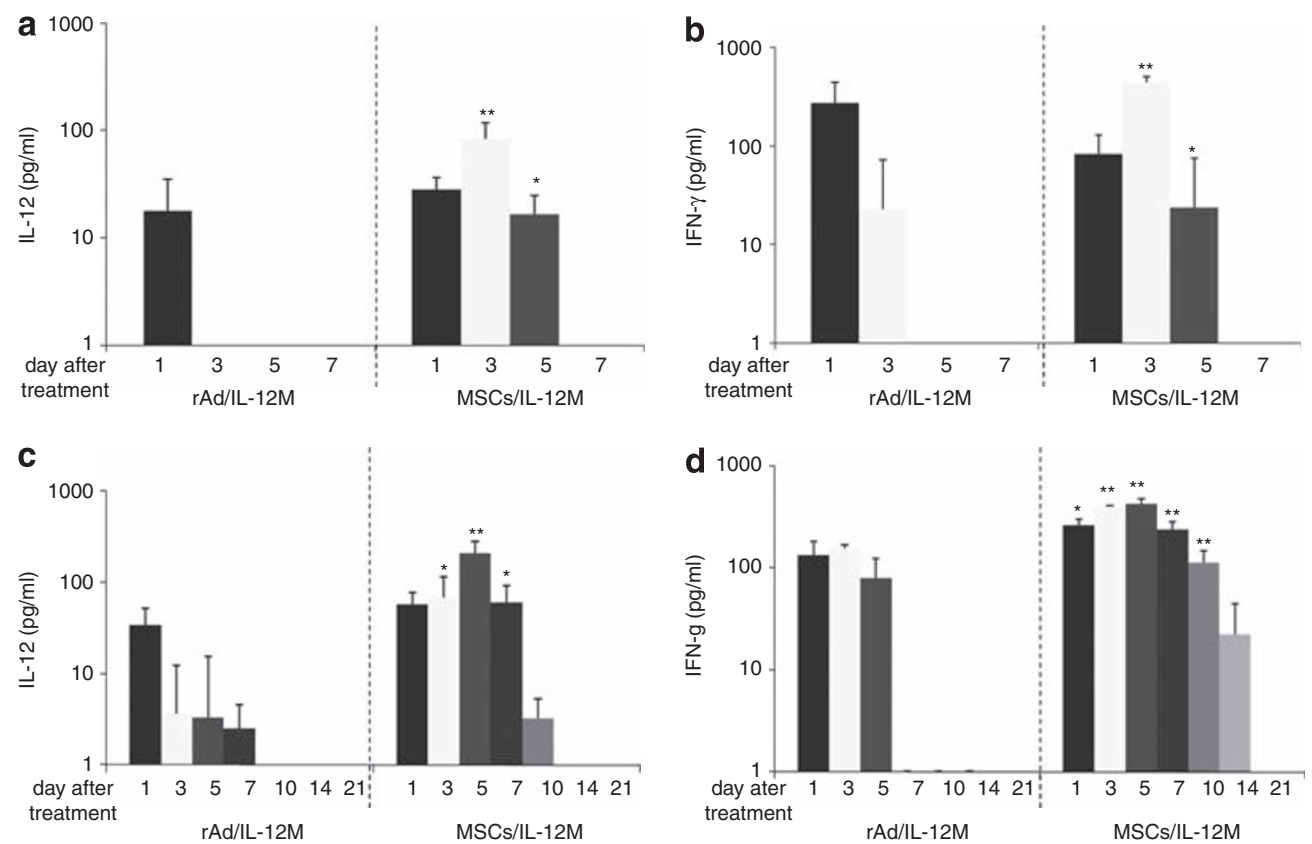

Figure 2 Systemic and local cytokine expression levels at various time points by mesenchymal stem cells (MSCs) expressing modified interleukin-12 (MSCs/ IL-12M) or rAd/IL-12M injection. Mice were injected with B16F10 s.c. and i.v. as described in Figure 1 legend. On day 1 after i.v. injection, mice were injected with $5 \times 10^{6}$ p.f.u. of $\mathrm{rAd} / \mathrm{IL}-12 \mathrm{M}, 1 \times 10^{5} \mathrm{MSCs}$ or MSCs/IL-12M. At the indicated time points, IL-12 and interferon (IFN)- $\gamma$ levels were determined both in serum (a, b) and the tumor (c, d) by ELISA. Negative control (omitted in graph) showed background level of cytokines. These results are representative of three independent experiments. ${ }^{*} P<0.05$ and ${ }^{*} P<0.01$. 
A

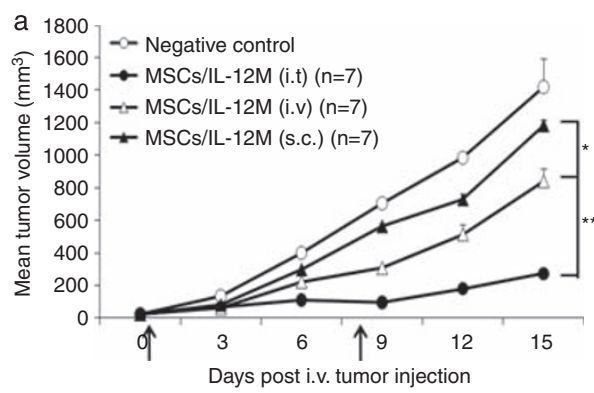

B

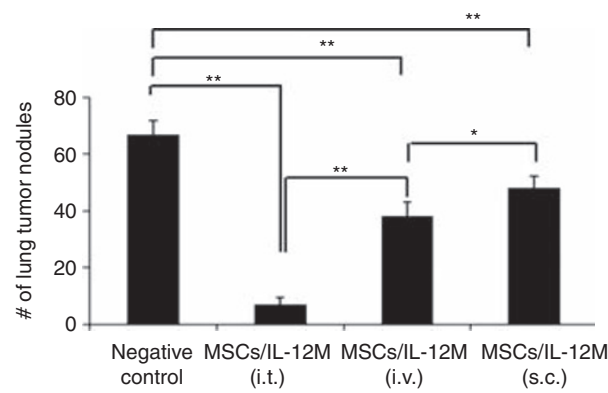

D

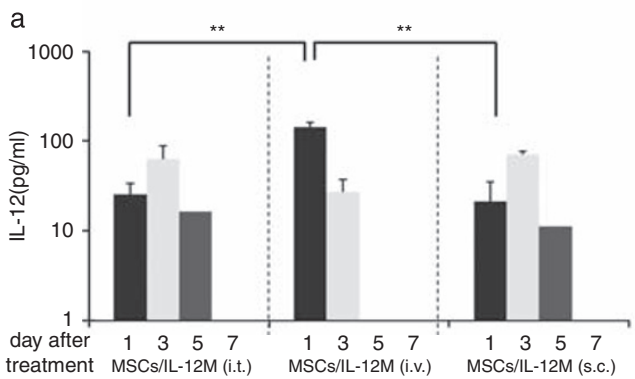

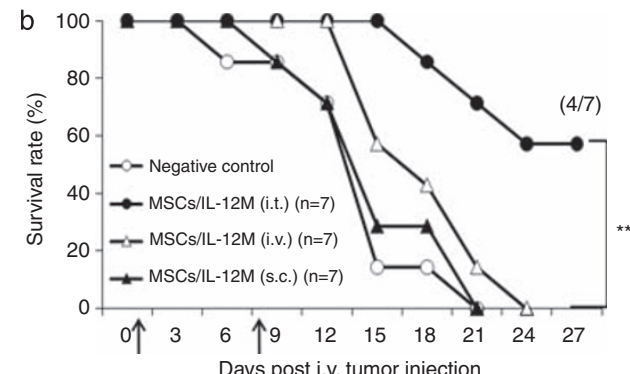

C
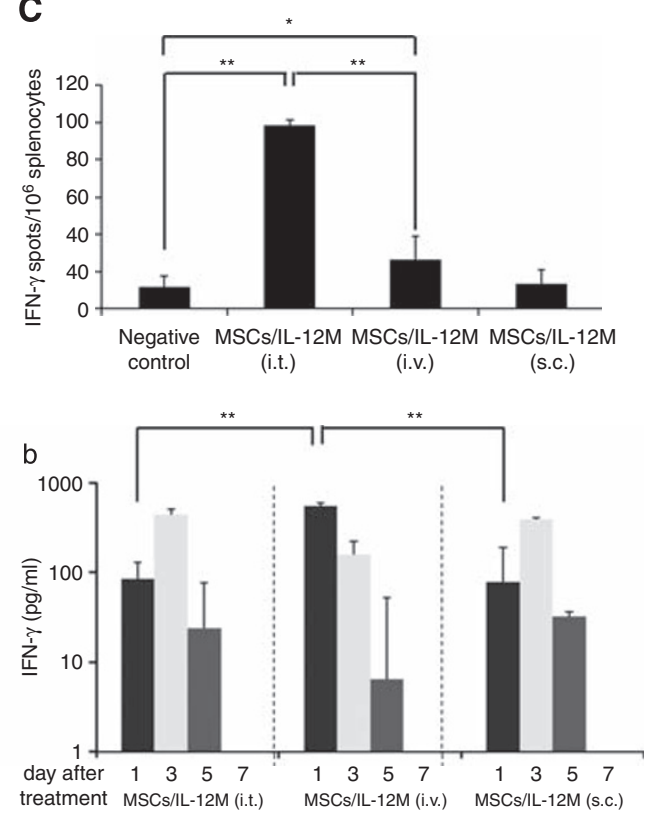

Figure 3 The effects of injection route on antitumor effects and level of cytokines induced by mesenchymal stem cells (MSCs) expressing modified interleukin-12 (MSCs/IL-12M) administration. Solid and metastatic tumors were established as described in Figure 1 legend. Mice were injected with $1 \times 10^{5}$ MSCs/IL-12M via intratumoral (i.t.), intravenous (i.v.) or subcutaneous (s.c.) route 1 and 8 days post i.v. tumor injection as indicated by arrows. Assessment of tumor mass (A-a), survival rates (A-b), lung metastases (B) and enzyme-linked immunosorbent spot (ELISPOT) assays (C) were performed as described in Figure 1 legend. (D) At the indicated time points, the serum levels of IL-12 (a) and interferon (IFN)- $\gamma$ (b) in B16F10 tumor-bearing mice were measured by ELISA. Background levels of cytokines were detected in negative control group (exclude from graph). This result was reproduced in three independent experiments. ${ }^{*} P<0.05$ and ${ }^{*} P<0.01$.

Matrigel embedding enhanced antitumor activity of MSCs/IL-12M, which was detectable even in immunodeficient mice

As Matrigel embedding was reported to enhance antitumor activities of MSCs/IL-12 against solid tumor growth in both the 4T1 breast cancer and B16F10 melanoma models, ${ }^{33}$ we examined the effect of Matrigel embedding on the antitumor activity of MSCs/IL-12M in mice bearing solid and metastatic tumor. As expected, MSCs/IL-12M embedded in Matrigel (MSCs/IL-12M/Matrigel) appeared to retard solid tumor growth and improve survival rate (Figure $4 \mathrm{a}, \mathrm{b}$ ). In addition, the number of lung tumor nodules was significantly reduced by Matrigel embedding (Figure 4c). It is worth noting that similar pattern was observed in growth inhibition of both types of tumors including solid and metastatic tumors. Overall, these antitumor effects were correlated with the magnitude of induced tumor-specific T-cell responses (Figure 4d), suggesting that induced T-cell responses appeared to have a major role in inhibiting metastatic and solid tumor growth. Consistent with the previous results, ${ }^{14}$ IL- 12 and IFN- $\gamma$ expression in serum and tumor site were increased and prolonged by the inclusion of Matrigel (Supplementary Figures 2a-d).
As NK and T cells are key players in immune-mediated antitumor effect, ${ }^{27,36}$ we evaluated antitumor effect of MSCs/IL-12M/Matrigel in several kinds of immunodeficient mice such as Beige (NK cell deficient), IFN- $\gamma$ knockout, $\mathrm{CD}^{+} \mathrm{T}$ cell-depleted, SCID and BNX (T, B and NK cell deficient) mice. As expected, antitumor effects by MSCs/IL-12M/Matrigel were not observed in IFN- $\gamma$ knockout mice (Figure $5 \mathrm{a}-\mathrm{c}$ ), which was consistent with a previous report that IL-12mediated antitumor effects were totally dependent on IFN- $\gamma \cdot{ }^{37}$ However, significant antitumor effects were observed in other immunodeficient mice, even in BNX mice, suggesting that other innate immunity such as macrophages and IFN- $\gamma$-induced anti-angiogenic effects might also contribute to antitumor effects observed here. It is likely that antitumor effects are more impaired in SCID and $\mathrm{CD}^{+}$ T-cell-depleted mice than in Beige mice $(P<0.05)$, indicating that $\mathrm{CD}^{+} \mathrm{T}$ cells have more roles in inhibiting tumor growth than NK cells in IL-12M-mediated antitumor activity. It is worth noting that the growth of solid tumor and the number of lung metastasis are significantly increased in BNX mice compared with other immunodeficient and wild-type mice, supporting the immunosurveillance 
a

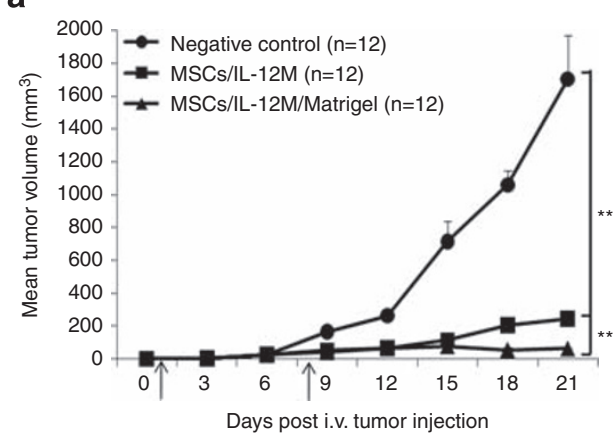

C

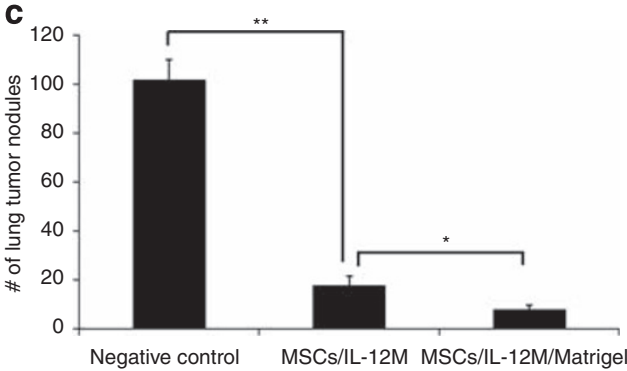

b
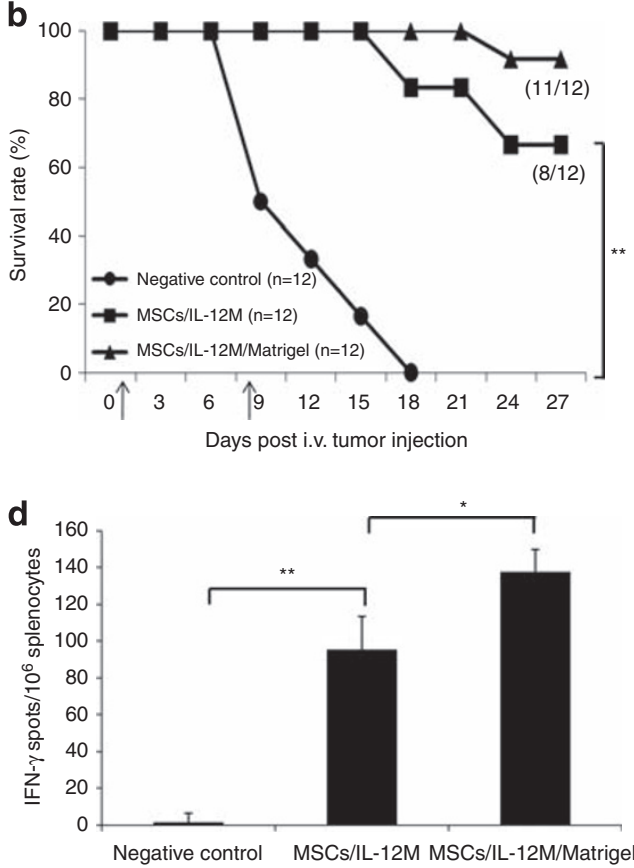

Figure 4 Effects of Matrigel embedding on the antitumor activities of mesenchymal stem cells (MSCs) expressing modified interleukin-12 (MSCs/LL-12M). Mice bearing solid and metastatic tumors were generated as described in Figure 1 legend and treated with MSCs/IL-12M in the presence or absence of Matrigel at the indicated time points by arrows. The treated mice were assessed for solid tumor growth (a), survival rates (b), lung metastases (c) and IFN- $\gamma$ production (d) using enzyme-linked immunosorbent spot (ELISPOT) assays as described in Figure 1 legend. Data are representative of three independent experiments. ${ }^{*} P<0.05$ and ${ }^{*} P<0.01$.

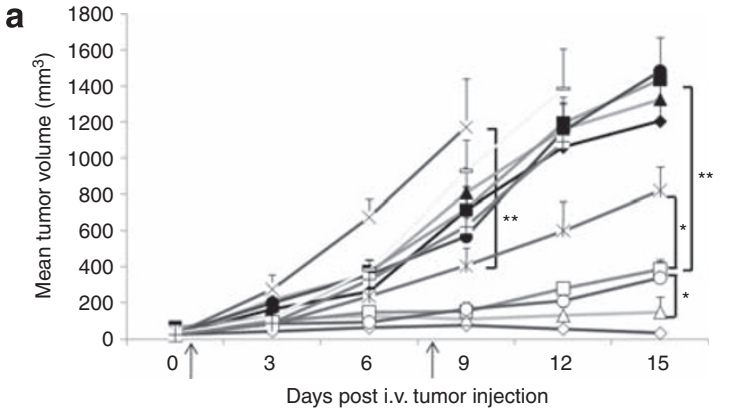

$\rightarrow$ C57BL/6 $(\mathrm{n}=12)$ No treatment

$-\infty$ C57BL/6 ( $n=12)$ MSCs.IL-12M/Matrigel

\- Beige $(\mathrm{n}=12)$ No treatment

$\triangle-$ Beige $(n=12)$ MSCs/L-12M/Matrigel

- CD8 depleted $(n=12)$ No treatment

$\rightarrow-$ CD8 depleted $(n=12)$ MSCs/LL-12M/Matrigel

$\rightarrow \operatorname{SCID}(n=12)$ No treatment

$\sim \operatorname{SCID}(n=12)$ MSCs/IL-12M/Matrigel

$-x-\operatorname{BNX}(n=12)$ No treatment

$-*-\operatorname{BNX}(n=12)$ MSCs/IL-12M/Matrigel

- IFN- $\gamma$ KO $(n=12)$ No treatment

-1- IFN- $\gamma$ KO ( $n=12)$ MSCs/IL-12M/Matrigel b

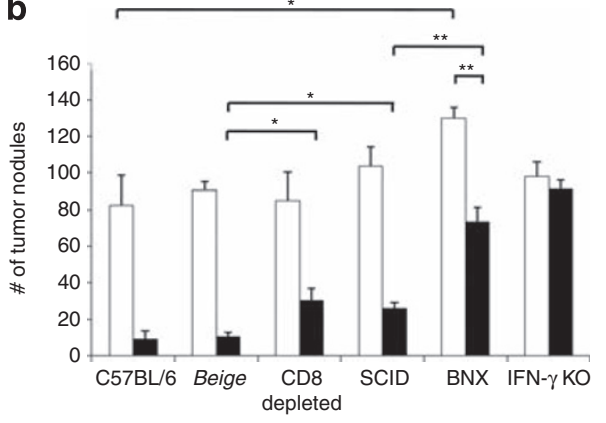

$\square$ No treatment

MSCs/IL-12M/Matrigel c

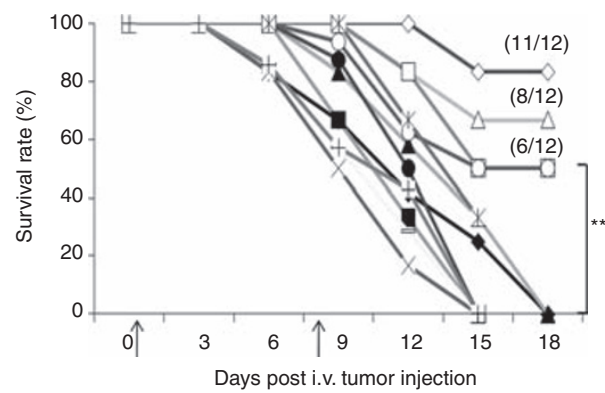

Figure 5 Antitumor activity of mesenchymal stem cells (MSCs) expressing modified interleukin-12 embedded in Matrigel (MSCs/LL-12M/Matrigel) in various immune cell-deficient mice. C57BL/6, Beige, CD8 ${ }^{+}$T-cell-depleted, BNX, SCID and interferon (IFN)- $\gamma$ knockout mice were injected with B16F10 tumor cells as described in Figure 1 legend. One day after i.v. B16F10 injection, mice were treated with MSCs/IL-12M/Matrigel twice as indicated by arrows. Depletion of $\mathrm{CD}^{+} \mathrm{T}$ cells was performed as described in materials and methods. The treated mice were assessed for tumor growth (a), number of lung metastases (b) and survival rates (c). Similar results were obtained in another independent experiment. ${ }^{*} P<0.05$ and ${ }^{* *} P<0.01$. 
theory that immune system continuously monitors its tissues for the presence of cancer cells as foreign invaders.

\section{DISCUSSION}

In this study, we evaluated relative effect of IL-12M-expressing adenovirus and MSCs on the capability of inducing tumor-specific $\mathrm{T}$-cell responses and antitumor effects by head-to-head comparison. Also, we used the same amount of adenovirus expressing IL-12M in ex vivo generation of MSCs/IL-12M to compare their relative antitumor activity quantitatively in mice bearing both solid and metastatic tumor, which may more closely mimic actual circumstances of patients with advanced tumor. Intratumoral injection of MSCs/ IL-12M conferred potent antitumor activities in inducing tumorspecific T-cell responses and inhibiting tumor growth at a greater degree than those of $\mathrm{rAd} / \mathrm{IL}-12 \mathrm{M}$, indicating that MSC is more effective than adenovirus as a cytokine gene delivery vehicle. These results are in part similar to the previous report that i.v. injection of MSCs/IL-12 exerted stronger antitumor activity than did rAd/IL-12. ${ }^{7}$

In this study, we used MSCs originated from rat bone marrow (xenogeneic). Previous report showed that injection of xenogenic MSCs can generate MSC-specific antibodies, which resulted in decreased population of MSCs at the injection site from $20 \%$ at day 2 to $2 \%$ at day $7 .{ }^{10}$ Therefore, syngeneic MSCs transduced with rAd/ IL-12M may induce stronger antitumor effect due to extended survival and more sustained secretion of IL-12 than rAd/IL-12M-transduced xenogeneic MSCs. However, there is safety concern on using syngeneic MSC because syngeneic MSCs can transform in vivo with repeated replication. ${ }^{38,39}$ In case of xenogeneic MSCs, tumorigenicity still remains undetermined.

Antitumor activity of MSCs/IL-12M regarding inhibition of solid and metastatic tumor growth as well as survival rate was further enhanced by Matrigel embedding, leading to induction of antitumor effects even in immunodeficient mice. These results are not consistent with previous report that IL-12-expressing MSCs embedded Matrigel did not show antitumor effect in NOD-SCID mice. ${ }^{33}$ This discrepancy may be caused by the different expression level due to enhanced transduction efficiency of rAd/IL-12M into MSCs by $4 \mathrm{HP} 4$ and the form of IL-12 used (IL-12 versus IL-12M). In addition, the difference of immunodeficient mice used and experimental condition may affect the discrepancy. As cancer patients normally show immunesuppressed condition, ${ }^{40}$ MSCs/IL-12M/Matrigel can be a therapeutic option to treat patients with immunodeficiency. It is worth noting that IL-12-responding cells include not only lymphoid cells but also non-lymphoid cells such as macrophages.

When we investigated the effects of injection route on the antitumor efficacy of MSCs/IL-12M using two different tumor models (B16F10 melanoma and TC-1 cervical cancer), MSCs/IL-12M injected via i.t. route induced tumor-specific T-cell responses more efficiently than did an i.v. or s.c. administration, leading to enhanced therapeutic efficacy against both solid and metastatic tumor. As MSCs were reported to have tumor tropism, ${ }^{41}$ MSCs/IL-12M injected via an i.v. route may migrate to the metastatic tumors and then inhibit their growth more efficiently than those injected via other routes (i.t. and s.c.). Surprisingly, we showed that i.t. injection of MSCs/IL-12M exhibited greater anti-metastatic activity than i.v. injection in mice bearing solid and metastatic tumor (Figure 3E and Supplementary Figure 1e, $P<0.05)$. This result indicated that tumor tropism of MSCs injected via an i.v. route is not strong enough to induce anti-metastatic effect compared with direct i.t. injection. Taken together, the injection route significantly affects the induction of systemic tumor-specific T-cell responses, which may be responsible for suppression of both metastatic and solid tumor growth in the context of IL-12-mediated antitumor effect. As i.v. injection of MSCs/IL-12M induced earlier and higher peak levels of cytokines than other routes and as there are no significant differences in cytokine expression levels and prolongation between s.c. and i.t. injection route, serum cytokine levels are unlikely to be an indicator of antitumor effects. Our result agrees with a previous observation that serum IL-12 levels were not correlated with antitumor effect, ${ }^{6}$ but does not agree with other several reports that serum cytokine level is proportional to antitumor potential in IL-12mediated tumor immunotherapy. ${ }^{42-44}$

Overall, action mechanism for inducing systemic tumor-specific T-cell responses which were responsible for regressing both solid and metastatic tumors may be mainly initiated with direct tumor cell killing by inflammatory cells and cytotoxic soluble factors recruited by cytokines at tumor site, resulting in the release of tumor antigens. These released tumor antigens should be loaded on professional antigen-presenting cells, especially dendritic cells, at tumor site as well as in the draining lymph node in order to present them to tumorspecific naïve $\mathrm{T}$ cells, which proliferate and differentiate into effector and memory T cells. Thus, the level and duration of cytokines such as IL-12 and IFN- $\gamma$ at tumor site, but not in serum, may have a major role in triggering tumor-specific T-cell responses. These tumor-specific effector T cells may migrate to recognize and destroy not only solid tumor but also metastatic tumor. It was reported that IL-12 expression at tumor site could attract activated lymphocytes by inducing intercellular adhesion molecule 1 and vascular cell adhesion protein 1 expression. ${ }^{45}$ In addition, IL-12 is critical for the development of peritumoral stroma required for accepting tumor-migrating T cells. ${ }^{46}$ Our findings provide a new insight for designing an optimal clinical protocol of cytokine gene-based immunotherapeutics for inducing not only the strongest tumor-specific T-cell responses but also antitumor effects.

\section{MATERIALS AND METHODS}

\section{IL-12M-expressing adenovirus}

rAd/IL-12M (rAd/IL-12N220L) was constructed as previously described ${ }^{5}$ using the AdEasy Vector System (QBiogene, Carlsbad, CA, USA).

\section{Preparation of genetically modified MSCs}

Bone marrow cells were collected from the femurs and tibias of 3- to 4-weekold SD rats by flushing respective tissues with Hank's balanced salts solution (WelGENE, Daegu, Korea) containing 2\% fetal bovine serum (Hyclone, Logan, UT, USA). After red blood cells were removed, bone marrow cells were filtered through a $40 \mu \mathrm{m}$ cell strainer (BD Bioscience, San Jose, CA, USA) and separated using Ficoll density gradient centrifugation. Isolated bone marrow cells were resuspended and cultured in Dulbecco's modified Eagle's medium $\left(1000 \mathrm{mgl}^{-1}\right.$ glucose; WelGENE) with $20 \%$ fetal bovine serum, $100 \mathrm{U} \mathrm{ml}^{-1}$ penicillin and $100 \mathrm{\mu g} \mathrm{ml}^{-1}$ of streptomycin (Invitrogen, Carlsbad, CA, USA) for about 10 days until colonies formed. Colonies were harvested and used for subsequent experiments as MSCs. MSCs were seeded into a $70 \mathrm{~mm}$ flask at an initial density of $1.4 \times 10^{6}$ cells and incubated overnight at $37^{\circ} \mathrm{C}$. The cells were infected with a mixture of rAd/IL-12M $\left(7 \times 10^{7}\right.$ p.f.u.) and $4 \mathrm{HP} 4$ (tetrameric form of HP4) $(0.1 \mu \mathrm{M})^{47}$ and incubated at $37^{\circ} \mathrm{C}$ for $30 \mathrm{~min}$. Cells were then trypsinized, washed with phosphate-buffered saline and injected into mice $\left(1 \times 10^{5}\right.$ cells per mouse).

\section{Cell lines}

B16F10 cells were purchased from the American Type Culture Collection (Manassas, VA, USA) and maintained in Dulbecco's modified Eagle's medium supplemented with $10 \%$ fetal bovine serum and $100 \mathrm{U} \mathrm{ml}^{-1}$ penicillin and $100 \mu \mathrm{g} \mathrm{ml}^{-1}$ streptomycin (Invitrogen). TC-1 cells were kindly provided by $\mathrm{Dr}$ TC Wu of Johns Hopkins University and maintained as previously described. ${ }^{48}$ 


\section{Mice}

Female C57BL/6 mice (6-8 weeks of age) were purchased from Charles River Breeding Laboratories (Shizuoka, Japan). Beige, BNX, IFN- $\gamma$ knockout and SCID mice were purchased from The Jackson Laboratory (Bar Harbor, ME, USA). All mice were maintained in the animal care facility at Pohang University of Science and Technology.

\section{Tumor establishment and treatment}

B16F10 cells $\left(1 \times 10^{6}\right)$ in $100 \mu$ l of phosphate-buffered saline were injected s.c. into the right hind flank of C57BL/6, Beige, BNX, IFN- $\gamma$ knockout and SCID mouse. When tumor diameters reached $5 \mathrm{~mm}$, mice were i.v. injected with $2 \times 10^{5} \mathrm{~B} 16 \mathrm{~F} 10$ cells in $100 \mu \mathrm{l}$ phosphate-buffered saline. At day 1 and 8 after i.v. tumor injection, C57BL/6 mice were injected in the tumors with $5 \times 10^{6}$ p.f.u. of $\mathrm{rAd} / \mathrm{IL}-12 \mathrm{M}, 1 \times 10^{5} \mathrm{MSCs}$ or MSCs/LL-12M in $100 \mu \mathrm{l}$ of Matrigel (BD Bioscience). Tumor growth and survival rate were monitored every 3 days. To assess the effects of the injection route, B16F10 tumor cells were injected as described above followed by injection of MSCs/IL-12M $\left(1 \times 10^{5}\right.$ cells $)$ via an i.t., i.v. or s.c. (contralateral to B16F10 implantation) route twice with 1-week interval. In the TC-1 tumor model, tumor injection and therapeutic treatment were performed as in the B16F10 melanoma model described above, except that $5 \times 10^{5}$ and $1 \times 10^{5}$ cells of TC- 1 were used for s.c. and i.v. injections, respectively. The number of lung metastases was assessed 14 days after i.v. tumor injection as previously described for all experiments. ${ }^{5}$

\section{In vivo $\mathrm{CD8}^{+} \mathrm{T}$-cell depletion}

Mice were intraperitoneally injected with rat anti-mouse CD8 monoclonal antibody prepared from the clone 2.43 at $500 \mu \mathrm{g}$ per mouse, starting at 1 day after i.v. tumor injection with 1-week intervals until the end of experiment. Depletion of $\mathrm{CD}^{+} \mathrm{T}$ cells was confirmed by fluorescence activated cell sorter analysis.

\section{Measurement of IL-12 and IFN- $\gamma$ expression levels}

Sera were collected from the orbital veins of tumor-bearing mice and the tumor mass was collected at the indicated time points after therapeutic injections. Tumor tissues were homogenized and protease inhibitor buffer (Roche Diagnostics, Mannheim, Germany) was added up to $500 \mu \mathrm{l}$. IL-12 and IFN- $\gamma$ levels were determined using the IL-12p70 and IFN- $\gamma$ ELISA kits (R\&D Systems, Minneapolis, $\mathrm{MN}$, USA), respectively, according to the manufacturer's instructions.

\section{IFN- $\gamma$ enzyme-linked immunosorbent spot assay}

Enzyme-linked immunosorbent spot assays were performed as previously described. ${ }^{5,48}$ Data were expressed as the mean \pm s.e.m. number of IFN- $\gamma$ secreting cells per $1 \times 10^{6}$ splenocytes.

\section{Statistical analysis}

All data were expressed as the mean \pm s.e.m. and were representative of at least two different experiments. Statistical difference between groups was assessed using a two-tailed Student's $t$-test. Statistical survival analysis was performed using the Kaplan-Meier method and log rank test. For all cases, differences were considered significant when $P$-values were $<0.05$.

\section{CONFLICT OF INTEREST}

The authors declare no conflict of interest.

\section{ACKNOWLEDGEMENTS}

We would like to thank Sang-Chun Lee, Kwan-Seok Lee and Bok-Chae Cho for their devoted animal care, Hong-Hwa Jun for technical assistance, and Dr Chang-Yul Kang for providing the clone 2.43. The Korean Healthcare Technology Research and Development Project, Ministry for Health, Welfare and Family Affairs, Republic of Korea, grant no. A091205-0911-0000300.

1 Choti MA. Chemotherapy-associated hepatotoxicity: do we need to be concerned? Ann Surg Oncol 2009; 16: 2391-2394.

2 Gottesman MM. Mechanisms of cancer drug resistance. Annu Rev Med 2002; 53 : 615-627.
3 Marshall E. Sciencescope. Science 1995; 268: 1555.

4 Car BD, Eng VM, Lipman JM, Anderson TD. The toxicology of interleukin-12: a review. Toxicol Pathol 1999; 27: 58-63.

5 Jin HT, Youn JI, Kim HJ, Lee JB, Ha SJ, Koh JS et al. Enhancement of interleukin-12 gene-based tumor immunotherapy by the reduced secretion of $\mathrm{p} 40$ subunit and the combination with farnesyltransferase inhibitor. Hum Gene Ther 2005; 16: 328-338.

6 Li S, Zhang L, Torrero M, Cannon M, Barret R. Administration route- and immune cell activation-dependent tumor eradication by IL12 electrotransfer. Mol Ther 2005; 12: 942-949.

7 Chen X, Lin X, Zhao J, Shi W, Zhang H, Wang Y et al. A tumor-selective biotherapy with prolonged impact on established metastases based on cytokine gene-engineered MSCs. Mol Ther 2008; 16: 749-756.

8 Bergelson JM, Cunningham JA, Droguett G, Kurt-Jones EA, Krithivas A, Hong JS et al. Isolation of a common receptor for Coxsackie B viruses and adenoviruses 2 and 5 . Science 1997; 275: 1320-1323.

9 Shiver JW, Emini EA. Recent advances in the development of HIV-1 vaccines using replication-incompetent adenovirus vectors. Annu Rev Med 2004; 55: 355-372.

10 Elzaouk L, Moelling K, Pavlovic J. Anti-tumor activity of mesenchymal stem cells producing IL-12 in a mouse melanoma model. Exp Dermatol 2006; 15: 865-874.

11 Pittenger MF, Mackay AM, Beck SC, Jaiswal RK, Douglas R, Mosca JD et al. Multilineage potential of adult human mesenchymal stem cells. Science 1999; 284: 143-147.

12 Friedenstein AJ, Piatetzky II S, Petrakova KV. Osteogenesis in transplants of bone marrow cells. J Embryol Exp Morphol 1966; 16: 381-390.

13 Barry FP, Murphy JM. Mesenchymal stem cells: clinical applications and biological characterization. Int J Biochem Cell Biol 2004; 36: 568-584.

14 Eliopoulos N, Al-Khaldi A, Crosato M, Lachapelle K, Galipeau J. A neovascularized organoid derived from retrovirally engineered bone marrow stroma leads to prolonged in vivo systemic delivery of erythropoietin in nonmyeloablated, immunocompetent mice. Gene Therapy 2003; 10: 478-489.

15 Glennie S, Soeiro I, Dyson PJ, Lam EW, Dazzi F. Bone marrow mesenchymal stem cells induce division arrest anergy of activated T cells. Blood 2005; 105: 2821-2827.

16 Studeny M, Marini FC, Dembinski JL, Zompetta C, Cabreira-Hansen M, Bekele BN et al. Mesenchymal stem cells: potential precursors for tumor stroma and targeteddelivery vehicles for anticancer agents. J Natl Cancer Inst 2004; 96: 1593-1603.

17 Ren C, Kumar S, Chanda D, Chen J, Mountz JD, Ponnazhagan S. Therapeutic potential of mesenchymal stem cells producing interferon-alpha in a mouse melanoma lung metastasis model. Stem Cells 2008; 26: 2332-2338.

18 Ren C, Kumar S, Chanda D, Kallman L, Chen J, Mountz JD et al. Cancer gene therapy using mesenchymal stem cells expressing interferon-beta in a mouse prostate cancer lung metastasis model. Gene Therapy 2008; 15: 1446-1453.

19 Loebinger MR, Eddaoudi A, Davies D, Janes SM. Mesenchymal stem cell delivery of TRAIL can eliminate metastatic cancer. Cancer Res 2009; 69: 4134-4142.

20 Gately MK, Renzetti LM, Magram J, Stern AS, Adorini L, Gubler U et al. The interleukin-12/interleukin-12-receptor system: role in normal and pathologic immune responses. Annu Rev Immunol 1998; 16: 495-521.

21 Drew PD, Lonergan M, Goldstein ME, Lampson LA, Ozato K, McFarlin DE. Regulation of MHC class I and beta 2-microglobulin gene expression in human neuronal cells. Factor binding to conserved cis-acting regulatory sequences correlates with expression of the genes. J Immunol 1993; 150: 3300-3310.

22 Dustin ML, Rothlein R, Bhan AK, Dinarello CA, Springer TA. Induction by IL 1 and interferon-gamma: tissue distribution, biochemistry, and function of a natural adherence molecule (ICAM-1). J Immunol 1986; 137: 245-254.

23 Sgadari C, Angiolillo AL, Tosato G. Inhibition of angiogenesis by interleukin-12 is mediated by the interferon-inducible protein 10. Blood 1996; 87: 3877-3882

24 Kanegane C, Sgadari C, Kanegane H, Teruya-Feldstein J, Yao L, Gupta G et al. Contribution of the CXC chemokines IP-10 and Mig to the antitumor effects of IL-12. J Leukoc Biol 1998; 64: 384-392.

25 Wang J, Murakami T, Hakamata Y, Ajiki T, Jinbu Y, Akasaka Y et al. Gene gun-mediated oral mucosal transfer of interleukin 12 cDNA coupled with an irradiated melanoma vaccine in a hamster model: successful treatment of oral melanoma and distant skin lesion. Cancer Gene Ther 2001; 8: 705-712.

26 Dranoff G. Cytokines in cancer pathogenesis and cancer therapy. Nat Rev Cancer 2004; 4: 11-22.

27 Colombo MP, Trinchieri G. Interleukin-12 in anti-tumor immunity and immunotherapy. Cytokine Growth Factor Rev 2002; 13: 155-168.

28 Gately MK, Carvajal DM, Connaughton SE, Gillessen S, Warrier RR, Kolinsky KD et al. Interleukin-12 antagonist activity of mouse interleukin-12 p40 homodimer in vitro and in vivo. Ann NY Acad Sci 1996; 795: 1-12.

$29 \mathrm{Ha}$ SJ, Chang J, Song MK, Suh YS, Jin HT, Lee CH et al. Engineering N-glycosylation mutations in IL-12 enhances sustained cytotoxic T lymphocyte responses for DNA immunization. Nat Biotechnol 2002; 20: 381-386.

30 Youn JI, Park SH, Jin HT, Lee CG, Seo SH, Song MY et al. Enhanced delivery efficiency of recombinant adenovirus into tumor and mesenchymal stem cells by a novel PTD. Cancer Gene Ther 2008; 15: 703-712.

31 Qiao L, Xu Z-L, Zhao T-J, Ye L-H, Zhang X-D. Dkk-1 secreted by mesenchymal stem cells inhibits growth of breast cancer cells via depression of Wnt signalling. Cancer Letters 2008; 269: 67-77.

32 Angoulvant D, Clerc A, Benchalal S, Galambrun C, Farre A, Bertrand Y et al. Human mesenchymal stem cells suppress induction of cytotoxic response to alloantigens. Biorheology 2004; 41: 469-476.

33 Eliopoulos N, Francois M, Boivin MN, Martineau D, Galipeau J. Neo-organoid of marrow mesenchymal stromal cells secreting interleukin-12 for breast cancer therapy. Cancer Res 2008; 68: 4810-4818. 
34 Fujii S, Shimizu K, Kronenberg M, Steinman RM. Prolonged IFN-gamma-producing NKT response induced with alpha-galactosylceramide-loaded DCs. Nat Immuno/ 2002; 3: 867-874.

35 Shi F, Rakhmilevich AL, Heise CP, Oshikawa K, Sondel PM, Yang NS et al. Intratumoral injection of interleukin-12 plasmid DNA, either naked or in complex with cationic lipid, results in similar tumor regression in a murine model. Mol Cancer Ther 2002; 1: 949-957.

36 Smyth MJ, Godfrey DI, Trapani JA. A fresh look at tumor immunosurveillance and immunotherapy. Nat Immunol 2001; 2: 293-299.

37 Yu WG, Ogawa M, Mu J, Umehara K, Tsujimura T, Fujiwara H et al. IL-12-induced tumor regression correlates with in situ activity of IFN-gamma produced by tumorinfiltrating cells and its secondary induction of anti-tumor pathways. J Leukoc Biol 1997; 62: 450-457.

38 Zhou YF, Bosch-Marce M, Okuyama H, Krishnamachary B, Kimura $\mathrm{H}$, Zhang $\mathrm{L}$ et al. Spontaneous transformation of cultured mouse bone marrow-derived stromal cells. Cancer Res 2006; 66: 10849-10854.

$39 \mathrm{Li} \mathrm{H}$, Fan X, Kovi RC, Jo Y, Moquin B, Konz R et al. Spontaneous expression of embryonic factors and p53 point mutations in aged mesenchymal stem cells: a model of age-related tumorigenesis in mice. Cancer Res 2007; 67: 10889-10898.

40 Marigo I, Dolcetti L, Serafini P, Zanovello P, Bronte V. Tumor-induced tolerance and immune suppression by myeloid derived suppressor cells. Immunol Rev 2008; 222 : 162-179.

41 Mishra PJ, Glod JW, Banerjee D. Mesenchymal stem cells: flip side of the coin. Cancer Res 2009; 69: 1255-1258.

42 Liu BY, Chen XH, Gu QL, Li JF, Yin HR, Zhu ZG et al. Antitumor effects of vaccine consisting of dendritic cells pulsed with tumor RNA from gastric cancer. World $\mathrm{J}$ Gastroenterol 2004; 10: 630-633.
43 Lee YS, Kim JH, Choi KJ, Choi IK, Kim H, Cho S et al. Enhanced antitumor effect of oncolytic adenovirus expressing interleukin-12 and B7-1 in an immunocompetent murine model. Clin Cancer Res 2006; 12: 5859-5868.

44 Kishida T, Asada H, Satoh E, Tanaka S, Shinya M, Hirai H et al. In vivo electroporationmediated transfer of interleukin-12 and interleukin-18 genes induces significant antitumor effects against melanoma in mice. Gene Therapy 2001; 8: 1234-1240.

45 Kanagawa N, Gao JQ, Motomura Y, Yanagawa T, Mukai Y, Yoshioka Y et al. Antitumor mechanism of intratumoral injection with IL-12-expressing adenoviral vector against IL-12-unresponsive tumor. Biochem Biophys Res Commun 2008; 372: 821-825.

46 Uekusa Y, Gao P, Yamaguchi N, Tomura M, Mukai T, Nakajima C et al. A role for endogenous IL-12 in tumor immunity: IL-12 is required for the acquisition of tumormigratory capacity by T cells and the development of T cell-accepting capacity in tumor masses. J Leukoc Biol 2002; 72: 864-873.

47 Park SH, Doh J, Park SI, Lim JY, Kim SM, Youn JI et al. Branched oligomerization of cell-permeable peptides markedly enhances the transduction efficiency of adenovirus into mesenchymal stem cells. Gene Therapy 2010; 17: 1052-1061.

48 Seo SH, Jin HT, Park SH, Youn JI, Sung YC. Optimal induction of HPV DNA vaccineinduced CD8+ T cell responses and therapeutic antitumor effect by antigen engineering and electroporation. Vaccine 2009; 27: 5906-5912.

This work is licensed under the Creative Commons Attribution-NonCommercial-No Derivative Works 3.0 Unported License. To view a copy of this license, visit http:// creativecommons.org/licenses/by-nc-nd/3.0/

Supplementary Information accompanies the paper on Gene Therapy website (http://www.nature.com/gt) 